\title{
A Two-Step Adaptive Error Recovery Scheme for Video Transmission over Wireless Networks
}

\author{
Daji Qiao and Kang G. Shin \\ Real-Time Computing Laboratory \\ Department of Electrical Engineering and Computer Science \\ The University of Michigan \\ Ann Arbor, Michigan 48109-2122 \\ E-mail: \{dqiao,kgshin\}@eecs.umich.edu
}

\begin{abstract}
In this paper, we investigate the transmission of $\mathbf{H . 2 6 3}$ video sequences over wireless networks with error recovery provided by a twostep adaptive hybrid ARQ scheme using RS codes. Each video frame is divided into data packets for transmission. For each packet transmission, by using a simple table-driven approach, the best RS code is selected from a given set of codes to minimize the transmission overhead. Further, an additional adaptation step is used to guarantee certain QoS requirement. Simulation results show that the proposed error recovery scheme outperforms the traditional single-code schemes and the single-step error recovery schemes thanks to its adaptability to both the wireless channel condition and the actual frame loss events.
\end{abstract}

Keywords - Wireless networks, adaptive hybrid ARQ, transmission gain, partial gain, transmission status, pseudo delivery deadline, frame loss rate.

\section{INTRODUCTION}

In recent years, the increasing number of mobile users and the growing demand for new multimedia services have spurred the research on video transmission over wireless networks. Unlike non-real-time message packets, real-time video frames have deadlines by which they must be delivered to the destination. Video services are very sensitive to delivery delays but can tolerate some frame losses and transmission errors. To provide a video service of acceptable quality over wireless networks, a carefully-designed error recovery scheme must be employed, because wireless channels are error-prone, bandwidth-limited and time-varying due to fading effects and user mobility.

There are two basic categories of error recovery: FEC (Forward Error Correction) and ARQ (Automatic Retransmission reQuest) [1], [2]. FEC schemes use error correcting codes to combat transmission errors by adding redundancy to data packets before they are transmitted. The redundancy is used by the receiver to detect and correct errors, if any. Because wireless channels are time-varying, stationary FEC schemes must be implemented to guarantee certain quality-of-service (QoS) for the worst case of the channel condition, which introduces a high transmission overhead. Since FEC schemes maintain constant throughput and bounded delay, they can be

The work reported in this paper was supported in part by the ONR under Grant N00014-99-1-0465. used for real-time applications. In contrast, only error detecting codes are used in ARQ schemes. Packets received in error are requested to be retransmitted. ARQ schemes are simple and efficient when the channel condition is good or moderate. If the channel bit error rate is high, the throughput performance drops drastically due to the increased frequency of retransmissions.

In order to achieve high throughput and high system reliability, a combination of these two basic error recovery schemes, called the hybrid ARQ scheme, has been proposed and received considerable attention. Deng [3] proposed a type-I hybrid ARQ system which automatically adjusts the error correcting code rate to match the current channel bit error rate. In [4], Kallel proposed and analyzed several efficient Stopand-Wait, Go-Back-N, and Select-Repeat hybrid ARQ protocols with adaptive forward error correction using convolutional codes. Joe [5] designed a hybrid ARQ scheme with concatenated FEC for wireless ATM networks, and the key idea is the adaptation of the code rate to channel conditions using incremental redundancy to maximize the throughput efficiency. However, for real-time applications, all the above error recovery schemes, proposed for non-real-time data transmission, may introduce an unacceptably long delay. To provide bounded delay, an error recovery scheme with a limited number of retransmissions has been proposed in [6]. Further, Liu [7] presented a delay-bounded type-II hybrid ARQ scheme using $\mathrm{BCH}$ codes for the transmission of $\mathrm{H} .263$ video sequences over indoor wireless channels. The number of retransmissions is limited to one in order to reduce the delay.

In this paper, we present a two-step adaptive hybrid ARQ scheme for transmitting H.263 video sequences which, (1) based on both the wireless channel condition and the deadline constraint, adaptively selects the best error correcting code by looking up an optimal code table which is pre-determined before starting the video service, and, (2) based on the actual frame loss events, adaptively uses the pre-built optimal code table to guarantee certain QoS in terms of the frame loss rate. This work is mainly motivated by [8] where a similar tabledriven approach is used to determine the best transmission 


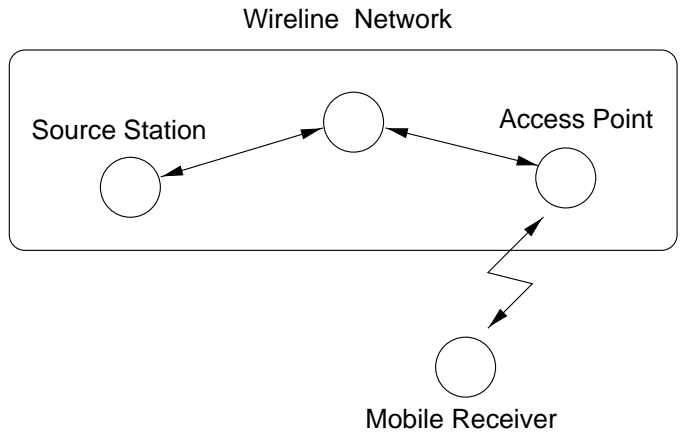

Fig. 1. Network topology

strategy with an objective of balancing the need of delivering the packet on time and the need to conserve bandwidth. However, this approach can't provide guaranteed QoS. In addition, they assume that each video frame is carried by a single packet for transmission, which is not practical.

The rest of this paper is organized as follows. Section 2 describes the system models and states the assumptions to be used. The problem statement and the proposed error recovery scheme are presented in Section 3. Section 4 presents and discusses the simulation results, and Section 5 concludes the paper.

\section{Modeling AND SPECIFICATIONS}

Before proceeding to the proposed two-step adaptive error recovery scheme, it is necessary to first describe the network topology, the wireless channel model, the H.263 video sequence, and the error correcting code.

\section{A. Network Topology}

As shown in Fig. 1, a mobile receiver and a source station communicate with each other via an access point (AP). Assume that the receiver station requests a video service from the source station where an H.263 video encoder is implemented with a fixed compression ratio. The picture format and the sample rate of the video sequence are known to both stations, and the AP buffers all the incoming packets from the source station. In this paper, we focus on the error recovery scheme at the AP for video transmission over the downlink of the endmost wireless hop. Error recovery for uplink communication will be treated in a separate paper.

\section{B. Wireless Channel Model}

The channel condition of a wireless link can be approximately modeled by a two-state Markov chain. The channel is in either good or bad state as shown in Fig. 2. Let $t_{i, j}$ denote the transition probability from state $i$ to state $j$, and let $P_{b}^{(s)}$ denote the average channel bit error rate at state $s$, where $i, j, s \in\{$ good, bad $\}$.

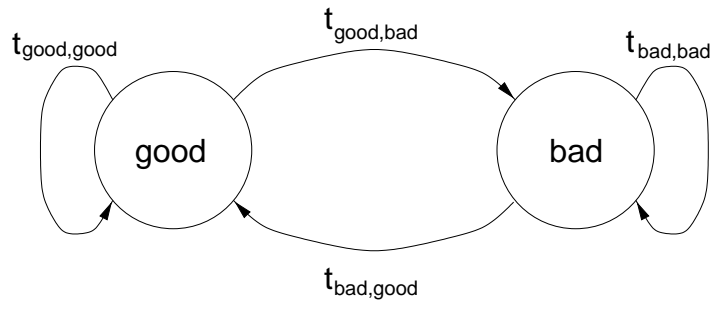

Fig. 2. Two-state Markov chain model for wireless channel

\section{H.263 Video Sequence Structure}

In the H.263 video coding standard [9], inter-frame prediction is employed to reduce the temporal redundancy between adjacent video frames. Each frame is first predicted from the immediately previous one in the same GOP (Group of Pictures), the motion vector and the residual prediction errors are then DCT (Discrete Cosine Transform) encoded. To accommodate the MTU (Maximum Transfer Unit) limitation of the underlying wireless network, the DCT coefficients are divided into smaller data packets for transmission. As shown in Fig. 3, a GOP consists of $L$ video frames. Let $\mathcal{F}=\left\{f_{0}, f_{1}, \ldots, f_{L-1}\right\}$ denote the set of $L$ different GOP positions.

We define the delivery deadline of a video frame to be the time when the frame is expected to be displayed at the receiver station. A frame can be fully restored if all of its packets are successfully transmitted before the delivery deadline. However, if any packet misses the delivery deadline, the reconstructed frame will contain some error and the picture quality will be degraded. Due to inter-frame prediction, the subsequent frames will also suffer quality degradation, and the error propagation won't stop until the first frame of the next GOP. These deteriorated frames are said to be lost. Instead of transmitting the packets of the subsequent lost frames, the AP stops the transmission and removes these packets from its memory.

Clearly, the number of lost frames due to packet losses depends on the GOP position of the frame carried in lost packets. To quantify the positive impact on the quality of service as the result of delivering the frame on time, each frame is associated with a reward value according to its GOP position. Consider a frame with GOP position $f_{i} \in \mathcal{F}$, any loss of its data packets

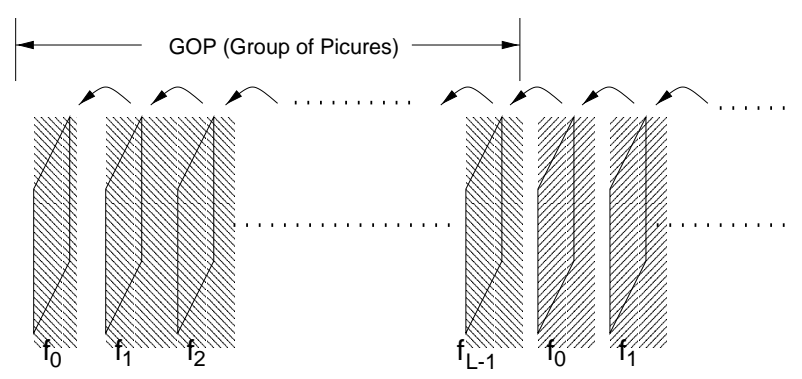

Fig. 3. H.263 video sequence structure 
would result in a total of $L-i$ lost frames. Based on this observation, we set the associated reward $R w d\left(f_{i}\right)$ to be $J \cdot(L-i)$, where $J$ is the number of data packets per frame.

\section{Error Recovery Scheme}

We consider an adaptive hybrid ARQ scheme using ReedSolomon (RS) error correcting codes. Instead of using one single RS code, a set of RS codes with different code rates are available at both the AP and the receiver station. At each attempt of packet transmission, the AP can use any one of these RS codes. In this paper, we consider the Stop-and-Wait ARQ strategy. The packet transmission is successful if a positive acknowledgment is received from the receiver station. If a negative acknowledgment is received or the acknowledgment is not received within a time-out interval, the packet is then retransmitted by the AP. The retransmission time-out interval is determined based on the round trip time. After a successful packet transmission or after the corresponding frame delivery deadline expires, the AP removes the packet from its memory.

RS codes are known to have the maximum error correcting capability for given redundancy. Let $c \equiv(N, K)$ be the RS code under consideration and let $q$ be the number of bits in each symbol. The error correcting capability $t$ is equal to $\lfloor(N-K) / 2\rfloor$, i.e., any combination of $t$ symbol errors out of $N$ symbols is correctable. The code rate $r$ is defined as $r=K / N$. Assume that the wireless channel is in state $s$ with the average channel bit error rate $P_{b}^{(s)}$. Let $P_{\text {sym }}$ denote the symbol error probability, then the probability of a correctable packet transmission, $P_{c o r}^{(s, c)}$, is given by

$$
P_{c o r}^{(s, c)}=\sum_{i=0}^{t}\left(\begin{array}{c}
N \\
i
\end{array}\right)\left(P_{s y m}\right)^{i}\left(1-P_{s y m}\right)^{N-i}
$$

where

$$
P_{\text {sym }}=1-\left(1-P_{b}^{(s)}\right)^{q}
$$

Let $\mathcal{C}=\left\{c_{0}, c_{1}, \ldots, c_{b}\right\}$ be the set of RS codes with code rates $r_{0}>r_{1}>\cdots>r_{b}>0$. For each $c_{i} \in \mathcal{C}$, let $C$ st $\left(c_{i}\right)$ denote the cost for using code $c_{i}$ in the packet transmission. When a packet of $h$ symbols is encoded by code $c_{i}$, the total number of symbols to be transmitted is $h / r_{i}$. Based on this observation, we set the associated transmission cost $C$ st $\left(c_{i}\right)$ to be $1 / r_{i}$. Clearly, for a fixed-length data packet, the larger the transmission cost, the better the error correcting capability. In the proposed approach, the AP can also choose to defer the packet transmission. For the sake of convenience, we use a special code $c_{0} \in \mathcal{C}$ with an infinite code rate to represent the decision of deferment. Choosing code $c_{0}$ makes the associated transmission cost zero, but, the probability of a correctable packet transmission is also zero.

\section{Problem Statement And Solutions}

Assume that the mobile receiver requests an H.263 video service from the source station and indicates its desired quality of service by setting a target frame loss rate ( $\left.F L R_{\text {target }}\right)$ of the received video sequence. In this paper, we define the transmission gain for the video frame under delivery as the difference between the associated reward for delivering the frame on time and the total transmission cost in conveying all the data packets of the frame. The problem is to develop an adaptive scheme for the AP to select the best RS code for the next packet transmission so as to maximize the expected transmission gain for the current frame, while keeping the actual frame loss rate below $F L R_{\text {target }}$.

\section{A. Adaptive RS Code Selection}

Our problem is actually an optimization problem with an additional constraint. Let's first deal with the gain maximization problem without considering the frame loss rate constraint.

The total transmission cost for the current frame delivery can be divided into two parts: the cost of past transmission attempts, and the future transmission cost in conveying the remaining packets of the frame. Since the cost of past transmission attempts has been determined and hence independent of the future transmission strategy, in order to maximize the expected transmission gain for the current frame, it is equivalent to maximizing the expected value of the difference between the associated reward and the future transmission cost, which is defined as the partial gain.

The transmission status at time $t$ can be represented by a quadruplet $(s, f, n, m)$, where $s \in\{$ good, bad $\}$ is the channel state in the Markov chain model, $f \in \mathcal{F}$ is the GOP position of the frame under delivery, $n$ is the number of the remaining packets of this frame, and $m$ is the number of packet transmission slots before the frame delivery deadline. In this paper, a packet transmission slot $(T)$ is defined as the time period from the beginning of a packet transmission until the packet retransmission time-out. Let $D$ denote the delivery deadline of the current frame, then $m$ can be calculated by

$$
m=\left\lfloor\frac{\max (D-t, 0)}{T}\right\rfloor .
$$

Let $P G(c, s, f, n, m)$ denote the expected partial gain of choosing code $c \in \mathcal{C}$ for the next packet transmission under the current transmission status $(s, f, n, m)$. The optimal code $c^{*}(s, f, n, m)$ and the maximum expected partial gain $P G^{*}(s, f, n, m)$ are defined as

$$
c^{*}(s, f, n, m)=\arg \max _{c \in \mathcal{C}} P G(c, s, f, n, m),
$$

and

$$
P G^{*}(s, f, n, m)=P G\left(c^{*}, s, f, n, m\right) .
$$

When $n>m$, even if $m$ transmission attempts are all successful, the current frame still can't be fully recovered before its delivery deadline due to absence of the remaining $n-m$ packets. Hence, it makes no sense to continue transmitting these $n$ packets. Instead, we select code $c_{0}$ to defer the next 
packet transmission and thus maximize the expected partial gain to be zero. When $n=0$, since all the packets of the current frame have been successfully transmitted, the maximum expected partial gain $P G^{*}(s, f, 0, m)$ is equal to $\operatorname{Rwd}(f)$.

Now consider the general case when $0<n \leq m$. A packet is successfully transmitted only if the transmission errors are correctable. Upon receiving a positive acknowledgment from the receiver station, the number of the remaining packets is reduced to $n-1$ with $m-1$ possible transmission attempts allowed before the delivery deadline. If the packet transmission fails, the AP has to retransmit the packet after the retransmission time-out. The number of the remaining packets is still $n$ but at most $m-1$ possible transmission attempts are allowed before the delivery deadline. In both cases, the transmission cost for using code $c$ is $C s t(c)$. Based on the above observations, $P G(c, s, f, n, m)(0<n \leq m)$ can be recursively calculated by

$$
\begin{aligned}
& P G(c, s, f, n, m)= \\
& \quad P_{c o r}^{(s, c)}\left[\sum_{i \in\{\text { good,bad }\}} t_{s, i} \cdot P G^{*}(i, f, n-1, m-1)\right] \\
& \quad+\left[1-P_{c o r}^{(s, c)}\right]\left[\sum_{i \in\{\text { good,bad }\}} t_{s, i} \cdot P G^{*}(i, f, n, m-1)\right] \\
& \quad-C s t(c) .
\end{aligned}
$$

Treating the cases of $n>m$ and $n=0$ as two initial conditions, we fully specify the computation of the optimal RS code for the next packet transmission by (4), (5), and (6).

Since the number of possible transmission status is finite for any practical video service, the AP can compute off-line a table of optimal RS codes indexed by the status quadruplet $(s, f, n, m)$. At run-time, the AP estimates the channel condition, determines the current transmission status, and looks up this pre-built code table to select the optimal RS code for the next packet transmission. The problem of how to make an accurate estimation of the current channel condition is beyond the coverage of this paper. In our study, we assume that the AP can monitor the past channel condition variations and make an estimation which is accurate enough for code selection.

\section{B. Adaptive Use of the Code Table}

When the AP looks up the pre-built optimal code table to determine the RS code for the next packet transmission, the expected transmission gain for the video frame under delivery is maximized according to the above analysis. However, since the frame loss rate constraint is not taken into consideration when building the optimal code table, the actual frame loss rate can't be automatically guaranteed below the target value. In this section, we propose an adaptive way of using the pre-built optimal code table to determine the best RS code for the next packet transmission, based on the observation of

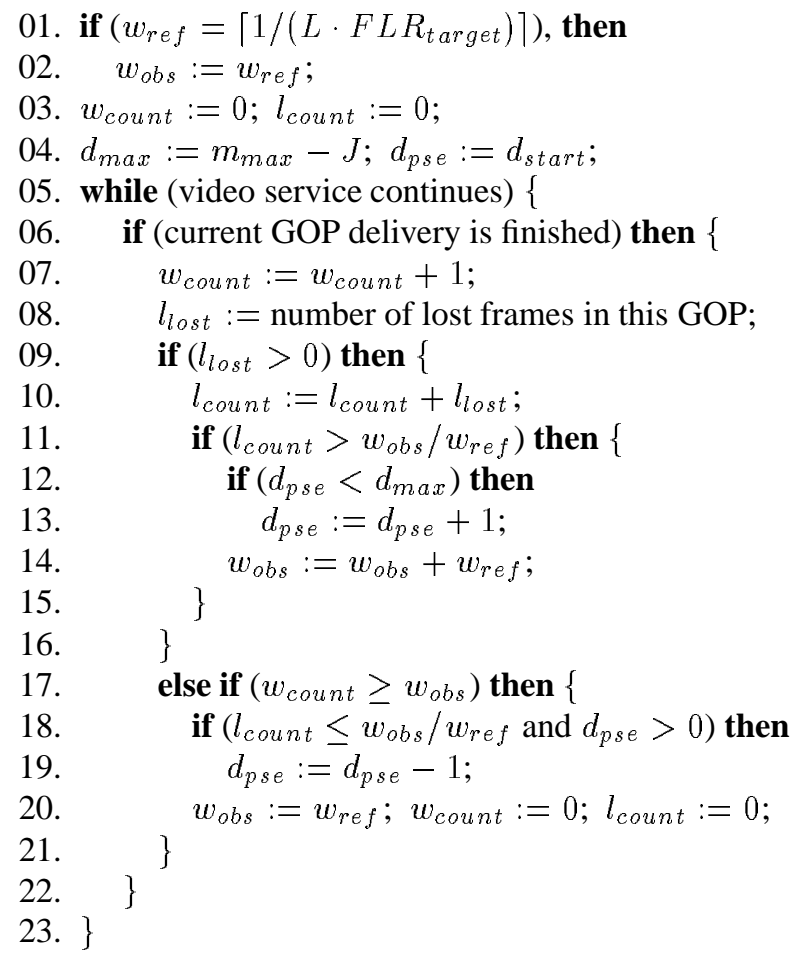

Fig. 4. A pseudo-code of the algorithm to adjust $d_{p s e}$

the actual frame loss events, so as to satisfy the frame loss rate constraint.

Consider two RS codes listed in the pre-built optimal code table: $c_{1}^{*} \equiv c^{*}\left(s, f, n, m_{1}\right)$ and $c_{2}^{*} \equiv c^{*}\left(s, f, n, m_{2}\right)$ with $m_{1}<m_{2}$. Note that $n$ and $m$ represent the number of the remaining packets of the current frame and the number of allowed transmission attempts before the delivery deadline, respectively. To transmit the same number $(n)$ of packets with a smaller $\left(m_{1}\right)$ number of transmission attempts, code $c_{1}^{*}$ tends to have lower code rate and higher error correcting capability than code $c_{2}^{*}$. Assume that the current transmission status is $\left(s, f, n, m_{2}\right)$. By choosing code $c_{1}^{*}$ over code $c_{2}^{*}$ for the next packet transmission, the AP offers more error protection, however, at the expense of lowering the expected transmission gain for the current frame.

Based on the above observations, we associate an earlier pseudo delivery deadline for each video frame, and let the AP determine the optimal transmission strategy based on this pseudo delivery deadline instead of the actual one. By changing a frame's pseudo delivery deadline, the AP adapts the transmission strategy with more or less error correcting capability. Let $d_{p s e}$ denote the number of packet transmission slots between the pseudo delivery deadline and the actual delivery deadline. Fig. 4 shows the pseudo-coded algorithm executed by the AP to adjust the value of $d_{p s e}$.

Before running the algorithm, the reference window size $w_{r e f}$ (measured in GOPs) is determined by $w_{r e f}=\lceil 1 /(L$. 
$\left.\left.F L R_{\text {target }}\right)\right\rceil$ and assigned to the observation window size $w_{\text {obs }}$, which represents the number of GOPs within which the AP counts the lost frames to estimate the actual frame loss rate $F L R_{\text {actual }}$. The counts $w_{\text {count }}$ for delivered GOPs and $l_{\text {count }}$ for lost frames within the observation window are reset to 0 , and $d_{p s e}$ is initialized to $d_{\text {start }}$, a design parameter. As shown in the pseudo-code, $w_{o b s}$ is increased or decreased by $w_{r e f}$, and the constraint $F L R_{\text {actual }} \leq F L R_{\text {target }}$ can be translated to that to keep the counted number $l_{\text {count }}$ of lost frames out of $w_{o b s}$ observed GOPs below $w_{o b s} / w_{\text {ref }}$. At run-time, whenever there is a frame loss after $w_{\text {obs }} / w_{\text {ref }}$ lost frames, $d_{p s e}:=d_{p s e}+1$ and $w_{o b s}:=w_{o b s}+w_{\text {ref }}$. On the other hand, when there are less than, or equal to, $w_{o b s} / w_{\text {ref }}$ lost frames out of $w_{o b s}$ observed GOPs, $d_{p s e}:=d_{p s e}-1$ and $w_{\text {obs }}:=w_{\text {ref }}$.

The maximum value of $d_{p s e}$ is $d_{\max }=m_{\max }-J$ in the algorithm. Note that $m_{\max }$, the maximum number of packet transmission slots for each frame delivery, is a constant determined by the picture format, the sample rate, and the compression ratio of the video sequence, and $J$ is the number of data packets per frame. Any $d_{p s e}$ with a value larger than $d_{\max }$ is meaningless because it is impossible to transmit $J$ packets in only $m_{\max }-d_{p s e}(<J)$ transmission attempts. We also set the minimum value of $d_{p s e}$ to 0 since a pseudo delivery deadline is never later than the actual delivery deadline.

After determining the value of $d_{p s e}$, the AP selects the best RS code for the next packet transmission by

$$
\begin{aligned}
& c^{* *}\left(s, f, n, m, d_{p s e}\right)= \\
& \begin{cases}c^{*}(s, f, n, m) & \text { if } d_{p s e}=0 \\
c^{*}\left(s, f, n, m-d_{p s e}\right) & \text { if } m-n>d_{p s e}>0 \\
c^{*}[s, f, n, \min (n, m)] & \text { if } d_{\text {max }} \geq d_{p s e} \geq m-n\end{cases} \\
& =c^{*}\left\{s, f, n, \min \left[m, \max \left(m-d_{p s e}, n\right)\right]\right\}
\end{aligned}
$$

where the quadruplet $(s, f, n, m)$ represents the current transmission status, and $c^{*}$ is the RS code listed in the pre-built optimal code table.

\section{Simulation And Results}

In this section, we evaluate the effectiveness of the proposed two-step adaptive error recovery scheme. The bandwidth of the wireless link is assumed to be $1 \mathrm{M}$ bps. For the results presented in this paper, let the state transition probabilities $t_{\text {good,bad }}$ and $t_{\text {bad,good }}$ be 0.2 and 0.8 , respectively, in the two-state Markov chain model of the wireless link.

The video under consideration is sampled at 20 frames/sec rate and each frame is captured in the QCIF format $(176 \times 144)$ with 256 gray levels for each pixel. At the source station, an H.263 video encoder with compression ratio 8.25 is implemented, and a single GOP consists of four video frames. We assume the data packet length to be 1024 bytes. Therefore, the compressed information of each video frame is carried by three data packets.

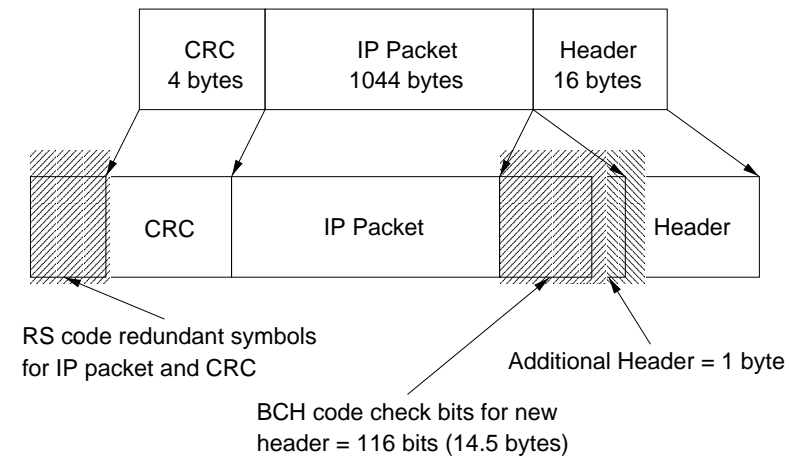

Fig. 5. Modified WaveLAN MAC frame structure

At the access point, each data packet is prepended with a 20 byte-long IP header and then encapsulated into a MAC frame for transmission. Our study borrows the MAC frame specification of the popular WaveLAN modem. In WaveLAN, the cyclic redundancy check (CRC) code is implemented for error detection, but no error correcting code is implemented. To apply the proposed adaptive error recovery scheme, the original WaveLAN MAC frame is modified as shown in Fig. 5.

The MAC header is increased by one byte, which is used to specify the selected RS code. The new MAC header is protected by a $(255,139)$ BCH binary code, so $255-139=116$ check bits are appended to the new header. By using this code, up to 15 bit errors can be corrected. The reason for choosing this code is addressed in [10]. IP packet and CRC check bits are encoded using the selected RS code, and the redundant symbols are appended at the end of the MAC frame. At the receiver station, based on the information provided in the new MAC header, the received MAC frames are then presented to the appropriate RS decoder.

Further, we assume that the three RS codes available at both the AP and the mobile receiver station are $c_{0}$ to defer the packet transmission, $c_{1} \equiv(919,839)$ and $c_{2} \equiv(939,839)$. Each symbol is 10 bits long $^{1}$. The $(919,839)$ RS code can detect and correct 40 symbol errors, while the $(939,839)$ RS code can detect and correct 50 symbol errors. The probabilities of a correctable packet transmission are computed by (1), using the average channel bit error rate of $5 \times 10^{-3}$ for bad state and $5 \times 10^{-6}$ for good state, respectively. These probabilities and the associated transmission cost for each RS code are listed in Table I.

For the assumed wireless link bandwidth of $1 \mathrm{M}$ bps, the transmission time of a data packet protected by code $c_{1}$ and code $c_{2}$ are $9.436 \mathrm{~ms}$ and $9.636 \mathrm{~ms}$, respectively. The packet transmission slot is set to $10 \mathrm{~ms}$, and hence the maximum number of packet transmission slots for each frame delivery is limited to five. The optimal RS code table indexed by the transmission status quadruplet $(s, f, n, m)$ is shown as Table II.

Empty entries in this optimal code table correspond to the

\footnotetext{
${ }^{1} 839$ symbols $\approx 1048$ bytes.
} 
TABLE I

THREE RS CODES UNDER CONSIDERATION

\begin{tabular}{|c|c|c|c|}
\hline RS code $c$ & $P_{\text {cor }}^{(\text {good }, c)}$ & $P_{\text {cor }}^{(\text {bad }, c)}$ & $C s t(c)$ \\
\hline \hline$c_{0}$ & 0 & 0 & 0 \\
\hline$c_{1}(919,839)$ & 1 & 0.253 & 1.095 \\
\hline$c_{2}(939,839)$ & 1 & 0.760 & 1.119 \\
\hline
\end{tabular}

TABLE II

PRE-BUILT OPTIMAL RS CODE TABLE

\begin{tabular}{|c|c|c|c|c|c|c|}
\hline$f$ & \multicolumn{5}{|c|}{$f_{0}$} \\
\hline$s$ & \multicolumn{3}{|c|}{ good } & \multicolumn{3}{c|}{$b a d$} \\
\hline \hline \multirow{3}{*}{$m$} & \multicolumn{3}{|c|}{$n$} & \multicolumn{3}{c|}{$n$} \\
\cline { 2 - 7 } & 1 & 2 & 3 & 1 & 2 & 3 \\
\hline 1 & $c_{1}$ & $c_{0}$ & $c_{0}$ & $c_{2}$ & $c_{0}$ & $c_{0}$ \\
\hline 2 & $c_{1}$ & $c_{1}$ & $c_{0}$ & $c_{2}$ & $c_{2}$ & $c_{0}$ \\
\hline 3 & $c_{1}$ & $c_{1}$ & $c_{1}$ & $c_{0}$ & $c_{2}$ & $c_{2}$ \\
\hline 4 & & $c_{1}$ & $c_{1}$ & & $c_{0}$ & $c_{2}$ \\
\hline 5 & & & $c_{1}$ & & & $c_{0}$ \\
\hline
\end{tabular}

\begin{tabular}{|c|c|c|c|c|c|c|}
\hline$f$ & \multicolumn{5}{|c|}{$f_{1}$} \\
\hline$s$ & \multicolumn{3}{|c|}{ good } & \multicolumn{3}{c|}{ bad } \\
\hline \hline \multirow{3}{*}{$m$} & \multicolumn{3}{|c|}{$n$} & \multicolumn{3}{c|}{$n$} \\
\cline { 2 - 7 } & 1 & 2 & 3 & 1 & 2 & 3 \\
\hline 1 & $c_{1}$ & $c_{0}$ & $c_{0}$ & $c_{2}$ & $c_{0}$ & $c_{0}$ \\
\hline 2 & $c_{1}$ & $c_{1}$ & $c_{0}$ & $c_{0}$ & $c_{2}$ & $c_{0}$ \\
\hline 3 & $c_{1}$ & $c_{1}$ & $c_{1}$ & $c_{0}$ & $c_{2}$ & $c_{2}$ \\
\hline 4 & & $c_{1}$ & $c_{1}$ & & $c_{0}$ & $c_{2}$ \\
\hline 5 & & & $c_{1}$ & & & $c_{0}$ \\
\hline
\end{tabular}

\begin{tabular}{|c|c|c|c|c|c|c|}
\hline$f$ & \multicolumn{5}{|c|}{$f_{2}$} \\
\hline$s$ & \multicolumn{3}{|c|}{ good } & \multicolumn{3}{c|}{ bad } \\
\hline \hline \multirow{3}{*}{$m$} & \multicolumn{3}{|c|}{$n$} & \multicolumn{3}{c|}{$n$} \\
\cline { 2 - 7 } & 1 & 2 & 3 & 1 & 2 & 3 \\
\hline 1 & $c_{1}$ & $c_{0}$ & $c_{0}$ & $c_{2}$ & $c_{0}$ & $c_{0}$ \\
\hline 2 & $c_{1}$ & $c_{1}$ & $c_{0}$ & $c_{0}$ & $c_{2}$ & $c_{0}$ \\
\hline 3 & $c_{1}$ & $c_{1}$ & $c_{1}$ & $c_{0}$ & $c_{0}$ & $c_{2}$ \\
\hline 4 & & $c_{1}$ & $c_{1}$ & & $c_{0}$ & $c_{2}$ \\
\hline 5 & & & $c_{1}$ & & & $c_{0}$ \\
\hline
\end{tabular}

\begin{tabular}{|c|c|c|c|c|c|c|}
\hline$f$ & \multicolumn{5}{|c|}{$f_{3}$} \\
\hline$s$ & \multicolumn{3}{|c|}{ good } & \multicolumn{3}{c|}{ bad } \\
\hline \hline \multirow{3}{*}{$m$} & \multicolumn{3}{|c|}{$n$} & \multicolumn{3}{c|}{$n$} \\
\cline { 2 - 7 } & 1 & 2 & 3 & 1 & 2 & 3 \\
\hline 1 & $c_{1}$ & $c_{0}$ & $c_{0}$ & $c_{2}$ & $c_{0}$ & $c_{0}$ \\
\hline 2 & $c_{1}$ & $c_{1}$ & $c_{0}$ & $c_{0}$ & $c_{2}$ & $c_{0}$ \\
\hline 3 & $c_{1}$ & $c_{1}$ & $c_{1}$ & $c_{0}$ & $c_{0}$ & $c_{2}$ \\
\hline 4 & & $c_{1}$ & $c_{1}$ & & $c_{0}$ & $c_{0}$ \\
\hline 5 & & & $c_{1}$ & & & $c_{0}$ \\
\hline
\end{tabular}
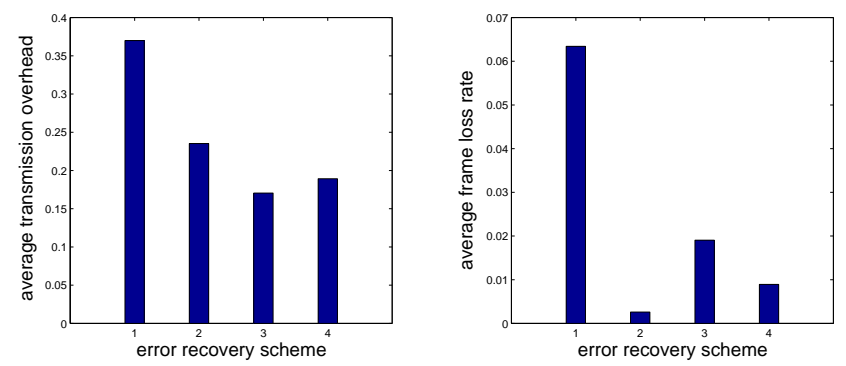

Fig. 6. Comparison of four error recovery schemes

events which never occur. For example, the transmission status with $(n=1, m=4)$ pair implies that $2(=3-n)$ data packets have been transmitted in only $1(=5-m)$ transmission attempt, which is impossible. Besides, the entry for transmission status with $n>m$ is always $c_{0}$, since it is impossible to transmit all the remaining packets before the frame delivery deadline.

For the transmission status with good state and $0<n \leq m$, the corresponding entry in the optimal code table is always $c_{1}$, since the probability of a correctable packet transmission is one for both $c_{1}$ and $c_{2}$, but $c_{1}$ has a smaller associated transmission cost. When the wireless link is in bad state, we have the following observations. The entry for $\left(b a d, f_{3}, 1,2\right)$ is $c_{0}$ or deferring the packet transmission at the current time. The rationale behind the deferment is that there is a high probability, 0.8 , that the wireless link will become good during the next packet transmission slot. However, if we change the GOP position from $f_{3}$ to $f_{0}$ in the quadruplet, the corresponding entry in the optimal code table indicates that, instead of deferring the transmission, the packet is encoded by code $c_{2}$ and transmitted over the bad channel. This is because failure to transmit frames with GOP position $f_{0}$ results in more subsequent frame losses than that for $f_{3}$, and we would like to provide more error protection and more transmission attempts for such frames. The rest of the entries for bad state can be interpreted similarly.

At each experiment, the mobile receiver requests a 60 second-long video service (total 1200 frames) from the source station, and indicates its desired quality of service by setting a target frame loss rate at $1.1 \%$. For each of the testing error recovery schemes, the experiment is repeated 100 times to estimate the average transmission overhead and the average frame loss rate of the video service. The four testing schemes under consideration are: the traditional single-code schemes using code $c_{1}$ (Scheme 1) and code $c_{2}$ (Scheme 2), a single-step error recovery scheme by simply looking up the pre-built optimal code table (Scheme 3), and the proposed two-step error recovery scheme (Scheme 4).

Fig. 6 shows the average transmission overhead and the average frame loss rate for each scheme. Scheme 2 has a large average transmission overhead because of the large number of check bits introduced by code $c_{2}$. However, due to these additional check bits, the receiver station can correct a large num- 

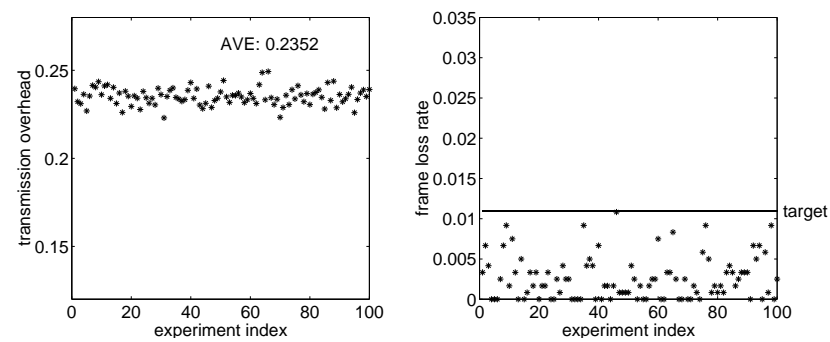

Fig. 7. Simulation results for Scheme 2

ber of symbol errors resulting in the lowest average frame loss rate. Scheme 1 has the highest average frame loss rate which is due to the low probability of correctable packet transmission using code $c_{1}$ when the wireless link is in bad state. Scheme 1 also has the largest average transmission overhead, because the poor error correcting capability of $\operatorname{code} c_{1}$ results in a large number of packet retransmissions. Scheme 3 has the smallest average transmission overhead among the four because of the adaptive use of code $c_{1}$ at good state and transmission deferment at bad state. It also yields a low average frame loss rate due to the added protection of code $c_{2}$.

The detailed simulation results for Scheme 2 are plotted in Fig. 7. Clearly, all the experiments meet the target frame loss rate requirement, however, at the expense of the large transmission overhead. As shown in Fig. 8, although Scheme 3 presents a relatively low average frame loss rate, most experiments still suffer the frame loss rate higher than the target value; this is because the optimal code table is built without considering the frame loss rate constraint. In the proposed two-step error recovery scheme, the pre-built optimal code table is adaptively
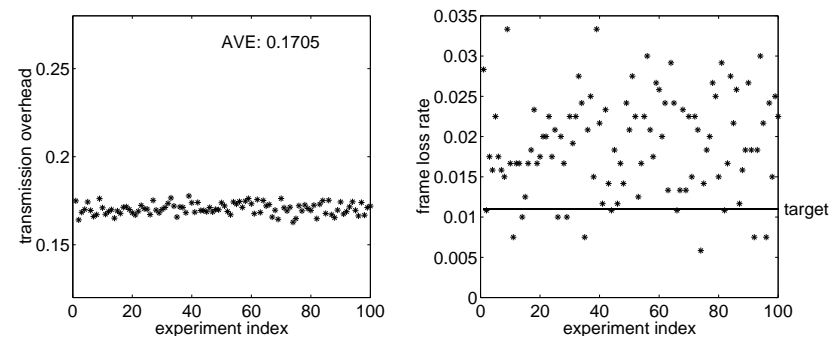

Fig. 8. Simulation results for Scheme 3
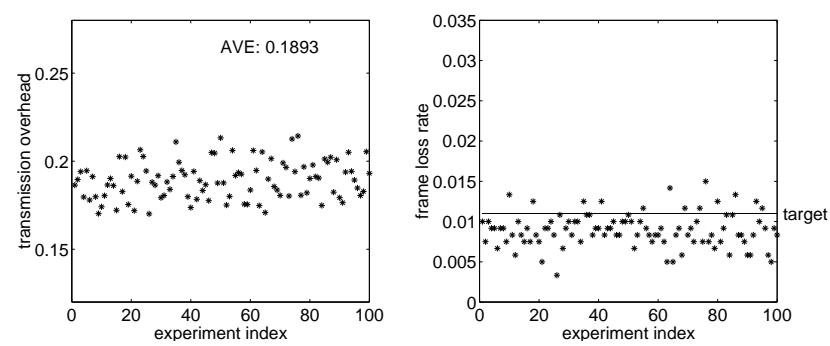

Fig. 9. Simulation results for Scheme 4 used according to the actual frame loss events. Due to this additional adaptation step, the frame loss rate is greatly improved at the expense of a slightly increased transmission overhead compared to Scheme 3. However, the average transmission overhead of the proposed scheme is almost $20 \%$ smaller than that of Scheme 2 because of the adaptive use of transmission deferment, codes $c_{1}$ and $c_{2}$ in each frame delivery. The results are shown in Fig. 6 and Fig. 9.

\section{CONCLUSION}

In this paper, we proposed a two-step adaptive error recovery scheme for video transmission over wireless networks. The mobile receiver indicates its desired quality of service by setting a target frame loss rate of the received video sequence. The main objective is to minimize the transmission overhead while keeping the frame loss rate below the target value. To accommodate the MTU limitation of the underlying wireless network, each video frame is divided into smaller data packets for transmission. The best RS code for the next packet transmission is carefully selected from a set of available codes based on the current transmission status and the observation of the actual frame loss events. Using simulations, we compare the performance of the proposed scheme with that of two traditional single-code schemes and a single-step adaptive scheme. The simulation results show that the proposed scheme is much better than the single-code schemes in terms of the transmission overhead and outperforms the single-step scheme in terms of the frame loss rate.

\section{REFERENCES}

[1] Shu Lin and Daniel J. Costello, Jr., Error Control Coding: Fundamentals and Applications, Prentice-Hall, Inc., Englewood, NJ, 1983.

[2] Hang Liu, Hairuo Ma, Magda El Zarki, and Sanjay Gupta, "Error control schemes for networks: An overview," Mobile Networks and Applications, vol. 2, no. 2, pp. 167-182, Oct. 1997.

[3] Robert H. Deng and Michael L. Lin, "A type I hybrid ARQ system with adaptive code rates," IEEE Transactions on Communications, vol. 43, no. 2/3/4, pp. 733-737, 1995.

[4] Samir Kallel, "Efficient hybrid ARQ protocols with adaptive forward error control," IEEE Transactions on Communications, vol. 42, no. 2/3/4, pp. 281-289, Mar. 1994.

[5] Inwhee Joe, "An adaptive hybrid ARQ scheme with concatenated FEC codes for wireless ATM," in Proceedings of ACM Mobicom'97, Budapest, Hungary, Sept. 1997.

[6] Qing Yang and Vijay K. Bhargava, "Delay and coding gain analysis of a truncated type-II hybrid ARQ protocol," IEEE Transactions on Vehicular Technology, vol. 42, no. 1, pp. 22-32, Feb. 1993.

[7] Hang Liu and Magda El Zarki, "Delay bounded type-II hybrid ARQ for video transmission over wireless channel," in Proceedings of Conference on Information Sciences and Systems, Princeton, NJ, Mar. 1996.

[8] Moncef Elaoud and Parameswaran Ramanathan, "Adaptive use of errorcorrecting codes for real-time communication in wireless networks," in Proceedings of IEEE Infocom'98, San Francisco, CA, Mar. 1998.

[9] Karel Rijkse, "H.263: Video coding for low-bit-rate communication," IEEE Communication Magazine, pp. 42-45, Dec. 1996.

[10] Sunghyun Choi and Kang G. Shin, "Cost-effective adaptive error control using a hybrid of FEC and ARQ in wireless networks," Tech. Rep. CSETR-402-99, University of Michigan, June 1999. 